\title{
Analysis of $\operatorname{Br}\left(\bar{B} \rightarrow X_{s} \gamma\right)$ at NNLO with a Cut on Photon Energy
}

\author{
Thomas Becher ${ }^{a}$ and Matthias Neubert ${ }^{b, c}$ \\ ${ }^{a}$ Fermi National Accelerator Laboratory, P.O. Box 500, Batavia, IL 60510, U.S.A. \\ ${ }^{b}$ Institute for High-Energy Phenomenology, Laboratory for \\ Elementary-Particle Physics, Cornell University, Ithaca, NY 14853, U.S.A. \\ ${ }^{c}$ Institut für Physik (ThEP), Johannes Gutenberg-Universität, D-55099 Mainz, Germany
}

(Dated: March 28, 2018)

\begin{abstract}
By combining a recent estimate of the total $\bar{B} \rightarrow X_{s} \gamma$ branching fraction at $O\left(\alpha_{s}^{2}\right)$ with a detailed analysis of the effects of a cut $E_{\gamma} \geq 1.6 \mathrm{GeV}$ on photon energy, a prediction for the partial $\bar{B} \rightarrow X_{s} \gamma$ branching fraction at next-to-next-to-leading order in renormalization-group improved perturbation theory is obtained, in which contributions from all relevant scales are properly factorized. The result $\operatorname{Br}\left(\bar{B} \rightarrow X_{s} \gamma\right)=(2.98 \pm 0.26) \cdot 10^{-4}$ is about $1.4 \sigma$ lower than the experimental world average. This opens a window for significant New Physics contributions in rare radiative $B$ decays.
\end{abstract}

\section{INTRODUCTION}

The inclusive decay $\bar{B} \rightarrow X_{s} \gamma$ is an important example of a flavor-changing neutral current process, which has been used to test the flavor sector of the Standard Model. Many groups have worked on improving the theoretical analysis of this process so as to keep pace with refinements in the measurements of its branching fraction. The effective weak Hamiltonian at next-to-next-toleading order (NNLO) has been obtained by calculating multi-loop matching coefficients and anomalous dimensions 1, 2, 3, 4]. While the fermionic NNLO corrections to the $b \rightarrow s \gamma$ matrix elements have been known for some time [5], complete NNLO corrections are presently only available for the electro-magnetic dipole operator [6, 7]. However, an approximate result for the NNLO charmpenguin contributions has just been published [8]. Combining these ingredients, a first estimate of the $\bar{B} \rightarrow X_{s} \gamma$ branching ratio at NNLO has been presented in $[9]$.

A complication in the analysis arises from the fact that measurements of the $\bar{B} \rightarrow X_{s} \gamma$ branching fraction impose stringent cuts on photon energy (defined in the $B$-meson rest frame), $E_{\gamma}>E_{0}$, with $E_{0}$ in the range between 1.8 to $2.0 \mathrm{GeV}$. The standard treatment is to extrapolate different measurements to a common reference point $E_{0}=1.6 \mathrm{GeV}$ using phenomenological models 10]. In that way, the experimental world average $\operatorname{Br}\left(\bar{B} \rightarrow X_{s} \gamma\right)=\left(3.55 \pm 0.24_{-0.10}^{+0.09} \pm 0.03\right) \cdot 10^{-4}$ has been derived [11]. The first error is statistical, the second one systematical, the third one is due to the extrapolation from high $E_{0}$ to the reference value, and the last error accounts for the subtraction of $\bar{B} \rightarrow X_{d} \gamma$ background. A theoretical result for the branching ratio with a cut at $E_{0}=1.6 \mathrm{GeV}$ has been derived in [9] using two-loop calculations of the photon-energy spectrum in fixed-order perturbation theory 12, 13. It has been argued that the extrapolation from the total to the partial branching fraction does not introduce additional theoretical uncertainties. This assertion is questionable because of the dynamical relevance of a soft scale $\Delta=m_{b}-2 E_{0} \approx 1.4 \mathrm{GeV}$, whose value is significantly lower than the $b$-quark mass.

Accounting for the photon-energy cut properly requires to disentangle contributions associated with the hard scale $\mu_{h} \sim m_{b}$, the soft scale $\mu_{0} \sim \Delta$, and an intermediate scale $\mu_{i} \sim \sqrt{m_{b} \Delta}$ set by the typical final-state hadronic invariant mass. When the cut value $E_{0}$ is chosen sufficiently low, $\Delta$ becomes a short-distance scale, and renormalization-group ( $\mathrm{RG}$ ) improved perturbation theory can be employed to calculate the effects of the photon-energy cut using a multi-scale operator product expansion [14]. We have recently performed a systematic analysis of these effects at NNLO. Two-loop corrections at the soft scale were calculated in 15], while those at the intermediate scale were computed in [16]. Here, the analysis is completed by extracting the two-loop hard matching corrections from a comparison with fixed-order calculations of the photon spectrum.

Using this method, we compute the fraction of all $\bar{B} \rightarrow X_{s} \gamma$ events with $E_{\gamma} \geq 1.6 \mathrm{GeV}$ with a perturbative precision of $5 \%$. At this level of accuracy several other, nonperturbative effects need to be evaluated carefully. The event fraction receives hadronic power corrections $\sim\left(\Lambda_{\mathrm{QCD}} / \Delta\right)^{n}$ governed by $B$-meson matrix elements of local operators. The leading correction $(n=2)$ is known and turns out to be small, but terms with $n \geq 3$ are presently unknown. Recently, a new class of enhanced $\Lambda_{\mathrm{QCD}} / m_{b}$ corrections to the $\bar{B} \rightarrow X_{s} \gamma$ decay rate has been identified, which involve matrix elements of nonlocal operators [17]. A model analysis using the vacuum insertion approximation indicates that these corrections affect the total decay rate at the level of a few percent.

Combining our result for the event fraction with the prediction for the total branching fraction from [8, 9], we obtain

$$
\operatorname{Br}\left(\bar{B} \rightarrow X_{s} \gamma\right)=(2.98 \pm 0.26) \cdot 10^{-4}
$$

for $E_{0}=1.6 \mathrm{GeV}$, where we have added in quadrature the uncertainties from higher-order perturbative effects $\left({ }_{-6}^{+4} \%\right)$, hadronic power corrections $(5 \%)$, parametric dependencies $(4 \%)$, and the interpolation in the charmquark mass $(3 \%)$. Two-loop perturbative corrections at the intermediate and soft scales significantly lower the branching fraction with regard to the fixed-order result given in [8], and they increase the theoretical uncertainty. 


\section{SCALE SEPARATION AND RESUMMATION}

At leading power in $\Lambda_{\mathrm{QCD}} / m_{b}$, the $\bar{B} \rightarrow X_{s} \gamma$ decay rate with a cut on photon energy obeys the factorization formula [18]

$$
\begin{aligned}
\Gamma\left(E_{0}\right)= & \frac{G_{F}^{2} \alpha}{32 \pi^{4}}\left|V_{t b} V_{t s}^{*}\right|^{2} \bar{m}_{b}^{2}(\mu)\left|H_{\gamma}(\mu)\right|^{2} \int_{0}^{\Delta} d p_{+}\left(m_{b}-p_{+}\right)^{3} \\
& \times \int_{0}^{p_{+}} d \omega m_{b} J\left(m_{b}\left(p_{+}-\omega\right), \mu\right) S(\omega, \mu)
\end{aligned}
$$

where $p_{+}=m_{b}-2 E_{\gamma}$ and $\Delta=m_{b}-2 E_{0}$. The function $H_{\gamma}$ contains hard quantum corrections, the jet function $J$ describes the physics of the hadronic final-state jet, and the shape function $S$ parameterizes bound-state effects inside the $B$-meson [19]. In the region of interest to our analysis, in which the quantity $\Delta \approx 1.4 \mathrm{GeV}$ can be treated as a perturbative scale, the double convolution integral in (2) can be evaluated using short-distance methods 14]. In order to isolate the effect of the photon cut, we focus on the fraction of all $\bar{B} \rightarrow X_{s} \gamma$ events that pass the cut $E_{\gamma} \geq E_{0}$, defined as $F\left(E_{0}\right)=\Gamma\left(E_{0}\right) / \Gamma(0)$. Since the total decay rate can be computed in fixed-order perturbation theory at the hard scale $\mu_{h}$, the event fraction obeys a factorization formula of the same form as (2), but with a different hard function $h$ in place of $\left|H_{\gamma}\right|^{2}$.

Contributions associated with different mass scales can be separated from each other by evolving the various objects in the factorization formula away from a common renormalization scale $\mu$ to different "matching scales", where they can be calculated reliably using fixed-order perturbation theory. In this process, single and double logarithms of ratios of the different scales are resummed to all orders. The matching scales should be taken close to the default values $\mu_{h}=m_{b}$ for $H_{\gamma}, \mu_{i}=\sqrt{m_{b} \Delta}$ for $J$, and $\mu_{0}=\Delta$ for $S$. An elegant expression describing the $\mathrm{RG}$ evolution of the jet function was derived in [20]. It involves an associated jet function $\widetilde{j}$, which is related to $J$ by a Laplace transform. The same technique can be applied to describe the evolution of the shape function in terms of an associated soft function $\widetilde{s}$. Inserting these results into (2), the integrations over $p_{+}$and $\omega$ can be performed, leading to

$$
\begin{aligned}
& F\left(E_{0}\right)=U\left(\mu_{h}, \mu_{i}, \mu_{0} ; \mu\right) \\
& \times\left(\frac{m_{b}}{\mu_{h}}\right)^{-2 a_{\Gamma}\left(\mu_{h}, \mu\right)}\left(\frac{m_{b} \Delta}{\mu_{i}^{2}}\right)^{2 a_{\Gamma}\left(\mu_{i}, \mu\right)}\left(\frac{\Delta}{\mu_{0}}\right)^{-2 a_{\Gamma}\left(\mu_{0}, \mu\right)} \\
& \times h\left(\frac{m_{b}}{\mu_{h}}\right) \tilde{j}\left(\ln \frac{m_{b} \Delta}{\mu_{i}^{2}}+\partial_{\eta}\right) \tilde{s}\left(\ln \frac{\Delta}{\mu_{0}}+\partial_{\eta}\right) \frac{e^{-\gamma_{E} \eta}}{\Gamma(1+\eta)} \\
& \times\left[p_{3}\left(\frac{\Delta}{m_{b}}\right)-\frac{\eta(1-\eta)}{6} \frac{\mu_{\pi}^{2}}{\Delta^{2}}+\ldots\right]+\delta F\left(E_{0}\right),
\end{aligned}
$$

where $\eta=2 a_{\Gamma}\left(\mu_{i}, \mu_{0}\right)>0$. Despite appearance, this result is independent of the choice of $\mu$ and of the three matching scales $\mu_{h}, \mu_{i}$, and $\mu_{0}$. For the special case $\mu=\mu_{i}$, relation (3) coincides with a formula derived previously in [21]. The terms in the first two lines arise from the $R G$ resummation of single and double logarithms. The precise form of the evolution factor $U$ will be given in 22]. The exponent

$$
a_{\Gamma}\left(\mu_{1}, \mu_{2}\right)=\int_{\mu_{1}}^{\mu_{2}} \frac{d \mu}{\mu} \Gamma_{\text {cusp }}\left(\alpha_{s}(\mu)\right)
$$

is an integral over the cusp anomalous dimension. The functions $h, \widetilde{j}$, and $\widetilde{s}$ in the third line contain the matching corrections at the hard, intermediate, and soft scales, respectively. The two-loop expression for the jet function $\widetilde{j}$ has been obtained in [16], while the two-loop result for the soft function $\widetilde{s}$ can be deduced from 15 . In the argument of these functions, $\partial_{\eta}$ means a derivative with respect to the quantity $\eta$. The hard function can be derived by matching (3) with the fixed-order expression for the photon spectrum derived in [12, 13]. At the default scale $\mu_{h}=m_{b}$, we find

$$
\begin{aligned}
h(1)= & +\frac{C_{F} \alpha_{s}}{4 \pi}\left[-\frac{52}{3}+\frac{7 \pi^{2}}{6}-\sum_{i \leq j} \operatorname{Re} \frac{C_{i}^{*} C_{j}}{\left|C_{7}\right|^{2}} \hat{f}_{i j}(1)\right] \\
& +C_{F}\left(\frac{\alpha_{s}}{4 \pi}\right)^{2}\left[C_{F} H_{F}+C_{A} H_{A}+T_{F} n_{f} H_{f}\right],
\end{aligned}
$$

where the term with $i=j=7$ is to be excluded from the sum. The one-loop result agrees with [14], and the two-loop coefficients are given by

$$
\begin{aligned}
& H_{F}=\frac{2297}{24}-\frac{229 \pi^{2}}{18}+\frac{89 \pi^{4}}{360}-22 \zeta_{3}+16 S_{\mathrm{a}}, \\
& H_{A}=-\frac{50521}{648}+\frac{1259 \pi^{2}}{108}-\frac{5 \pi^{4}}{18}+\frac{313}{9} \zeta_{3}+16 S_{\mathrm{na}}, \\
& H_{f}=\frac{9365}{162}-\frac{19 \pi^{2}}{27}-\frac{92}{9} \zeta_{3} .
\end{aligned}
$$

The constants $S_{\mathrm{a}} \approx 1.216$ and $S_{\mathrm{na}} \approx-4.795$ have been obtained by numerical integration in [12. The complete expression for $h\left(m_{b} / \mu_{h}\right)$ including scale dependence will be given in [22].

In the last line of (3), the hadronic quantity $\mu_{\pi}^{2}$ parameterizes the $B$-meson matrix element of the kinetic operator in heavy-quark effective theory. The ellipses represent unknown hadronic power corrections of order $\left(\Lambda_{\mathrm{QCD}} / \Delta\right)^{3}$. The polynomial

$$
p_{3}(\delta)=1-\frac{3 \delta \eta}{1+\eta}+\frac{3 \delta^{2} \eta}{2+\eta}-\frac{\delta^{3} \eta}{3+\eta}
$$

derives from the $\left(m_{b}-p_{+}\right)^{3}$ prefactor in (2). The term $\delta F\left(E_{0}\right)$ in (3) collects terms that are power suppressed in the ratio $\delta=\Delta / m_{b} \approx 0.3$. These corrections are known in fixed-order perturbation theory, but no resummation is available. We express them as a perturbative series in powers of $\alpha_{s}(\mu)$. The one-loop result for $\delta F$ has been given in [14], while the dominant two-loop contribution has recently been calculated in 12, 13]. For subleading contributions from other operators in the effective Hamiltonian only the fermionic two-loop corrections are known [23]; they have a negligible impact on our results. 

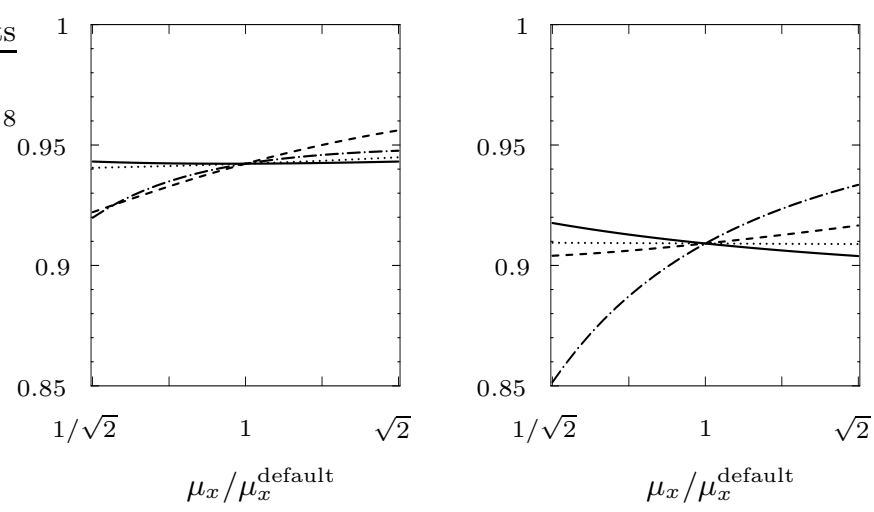

FIG. 1: Scale dependence of the ratio $T$. The curves show the effect of varying the hard scale $\mu_{h}$ (dotted), intermediate scale $\mu_{i}$ (dashed), soft scale $\mu_{0}$ (dash-dotted), and the reference scale $\mu$ (solid) by a factor of $\sqrt{2}$ about their default values. In the left plot the resummation is performed for the leadingpower terms only, while in the right plot the $p_{3}$ term is also included (see text for further explanation).

\section{RESULTS AND CONCLUSIONS}

In (31), the parameters $m_{b}$ and $\mu_{\pi}^{2}$ are defined in the on-shell scheme. To improve the perturbative behavior, one should eliminate them in favor of appropriately defined short-distance quantities. We use the "shapefunction scheme" proposed in 24], in which low-scale subtracted heavy-quark parameters are defined via the moments of the renormalized $B$-meson shape function, regularized with a hard cutoff $\mu_{f}$. The two-loop relations between the shape-function and pole-scheme parameters have been derived in 25]. We adopt the conventional choice $\mu_{f}=1.5 \mathrm{GeV}$ and use the values $m_{b}=$ $(4.61 \pm 0.06) \mathrm{GeV}$ and $\mu_{\pi}^{2}=(0.15 \pm 0.07) \mathrm{GeV}^{2}$ extracted from a fit to moments of inclusive $B$-decay spectra 21.

Ref. [8] does not provide a result for the total $\bar{B} \rightarrow X_{s} \gamma$ branching fraction. The most inclusive quantity considered is the partial branching fraction defined with a mild cut at $E_{0}=1 \mathrm{GeV}$. In order to combine this prediction with our RG-improved result for the event fraction, we define the ratio $T=F(1.6 \mathrm{GeV}) / F(1.0 \mathrm{GeV})$. We evaluate the various matching scales at their default values, namely $\mu_{h}=m_{b}, \mu_{0}=\Delta$, and $\mu_{i}=\mu=\sqrt{m_{b} \Delta}$, where the values of $\Delta$ are different in the numerator and denominator. In order to study the residual scale dependence, we then vary each of the four scales by a factor of $\sqrt{2}$, correlated between numerator and denominator. While the ratio $T$ is formally independent of these scales, the residual dependence of the truncated perturbative expression can be taken as an estimate of higher-order effects. The results are depicted in Figure 1 Not surprisingly, the dominant effect arises from varying the lowest scale, $\mu_{0} \sim \Delta$, while the scale variations at the intermediate and high scales have a lesser impact.

The term proportional to $p_{3}\left(\Delta / m_{b}\right)$ in (3) includes a subset of power corrections associated with a phase-space factor. While it was possible to perform the scale separa- tion for these terms, treating them in a different way than the remaining power corrections in $\delta F\left(E_{0}\right)$ is somewhat arbitrary. The right plot in the figure refers to the form of the factorization formula shown in (3), while the left plot corresponds to expanding out $\left(p_{3}-1\right)$ in fixed-order perturbation theory and including it in the $\delta F$ term. While the stability with respect to variations of the soft scale is better in this case, the perturbative corrections turn out to be smaller when performing the resummation for the $p_{3}$ term. The shift in central value between the two schemes is about $3 \%$, which is inside the error bar. This effect hints at the importance of $\mathrm{RG}$ resummation for the power corrections.

To quote our final result we take the average of the two schemes and assign an asymmetric error reflecting the scale variation. This yields

$$
T=0.93_{-0.05 \text { pert }}^{+0.03} \pm 0.02_{\text {hadr }} \pm 0.02_{\text {pars }} .
$$

The event fraction $F\left(E_{0}\right)$ receives hadronic power corrections not suppressed by inverse powers of $m_{b}$, but only by powers of the soft scale $\Delta$ [14]. These corrections are governed by $B$-meson matrix elements of local operators. The leading effect proportional to $\mu_{\pi}^{2}$ in (3) is small mainly due to the smallness of its coefficient $\eta(1-\eta) / 6$. Generically, we expect subleading corrections to scale like $\eta\left(\Lambda_{\mathrm{QCD}} / \Delta\right)^{3}$, for which we assign a $2 \%$ uncertainty. The main uncertainties from parameter variations are $\mp 0.9 \%$ for $\alpha_{s}\left(m_{Z}\right)=0.1189 \pm 0.0020$, $\pm 0.4 \%$ for $m_{c} / m_{b}=0.26 \pm 0.03$, and $\pm 0.1 \%$ for $m_{b}=$ $(4.61 \pm 0.06) \mathrm{GeV}$. We also include a variation of $\pm 1.2 \%$ due to the fact that the three-loop anomalous dimension of the shape function is yet unknown 22]. Our value for the ratio $T$ is lower than the estimate $T=0.963$ obtained in fixed-order perturbation theory [9]. Moreover, we find that there is a significant theoretical uncertainty inherent in the calculation of $T$.

In order to complete the analysis we need as input the theoretical result for the $\bar{B} \rightarrow X_{s} \gamma$ branching fraction with $E_{0}=1.0 \mathrm{GeV}$, which we take from the fixedorder NNLO calculation of [8, 9]. These authors find $\operatorname{Br}\left(\bar{B} \rightarrow X_{s} \gamma\right)=(3.27 \pm 0.23) \cdot 10^{-4}$ for $E_{0}=1 \mathrm{GeV}$, where the error has been obtained by adding in quadrature the uncertainties from higher-order perturbative corrections $(3 \%)$, nonperturbative effects $(5 \%)$, parameter dependencies $(3 \%)$, and the interpolation in the charmquark mass employed in the NNLO estimate of charmpenguin loop graphs (3\%). The $\bar{B} \rightarrow X_{s} \gamma$ branching fraction receives incalculable power corrections starting at order $\Lambda_{\mathrm{QCD}} / m_{b}$, which cannot be described using the operator product expansion [26]. While we disagree with [8] on the statement that these effects are suppressed by a power of $\alpha_{s}\left(m_{b}\right)$, we nevertheless believe that the corresponding uncertainty should not be larger than $5 \%$. Recently, a new class of enhanced power corrections has been identified [17]. At tree level their effects are parameterized in terms of $B$-meson matrix elements of nonlocal four-quark operators. Using the vacuum insertion approximation, a reduction of the total branching fraction 
between $0.3 \%$ and $3 \%$ has been found. Accounting for this effect lowers the central value from 3.27 to 3.22 .

Our final prediction for the $\bar{B} \rightarrow X_{s} \gamma$ branching fraction with $E_{0}=1.6 \mathrm{GeV}$ is

$$
\begin{aligned}
& \operatorname{Br}\left(\bar{B} \rightarrow X_{s} \gamma\right) \\
& =\left(2.98_{-0.17 \text { pert }}^{+0.13} \pm 0.16_{\text {hadr }} \pm 0.11_{\text {pars }} \pm 0.09_{m_{c}}\right) \cdot 10^{-4}
\end{aligned}
$$

where we have combined errors of the same type in quadrature. This appears justified, since theoretical correlations in the calculations of the total branching fraction and the event fraction $F\left(E_{0}\right)$ are small. In (1) we have combined all uncertainties in quadrature. A more conservative approach would be to add the errors linearly, in which case the error becomes ${ }_{-0.53}^{+0.49}$. Compared with the result $\operatorname{Br}\left(\bar{B} \rightarrow X_{s} \gamma\right)=(3.15 \pm 0.23) \cdot 10^{-4}$ obtained in [8], our central value in (9) is lower by about $5 \%$ and, more importantly, the perturbative uncertainty is larger by almost a factor of 2 . Both changes are a result of significant two-loop corrections encountered at the intermediate and soft scales, $\mu_{i} \sim \sqrt{m_{b} \Delta}$ and $\mu_{0} \sim \Delta$.

Our theoretical prediction for the $\bar{B} \rightarrow X_{s} \gamma$ decay rate is consistent with the present experimental world average, as is reflected in the ratio

$$
\frac{\operatorname{Br}\left(\bar{B} \rightarrow X_{s} \gamma\right)_{\text {exp }}}{\operatorname{Br}\left(\bar{B} \rightarrow X_{s} \gamma\right)_{\mathrm{th}}}=1.19 \pm 0.09_{\mathrm{exp}} \pm 0.10_{\mathrm{th}}
$$

However, whereas for a long time the experimental result used to be lower than the theoretical one, it is now about 1.4 standard deviations larger. Since in many extensions of the Standard Model the contributions from
New Physics are expected to interfere constructively with the Standard Model $b \rightarrow s \gamma$ amplitude, the situation has changed from one where New Physics models were rather tightly constrained to one where there is now room for speculation about how the central number in (10) could be explained in terms of loop contributions containing new heavy particles. Consider, e.g., the case of type-II two-Higgs-doublet models. Whereas in the past there used to be bounds on the charged-Higgs mass from $\bar{B} \rightarrow X_{s} \gamma$ on the order of $500 \mathrm{GeV}$, the ratio (10) could now be explained with a New Physics contribution from a charged Higgs in just that mass range.

In summary, we have presented the first NNLO prediction for the $\bar{B} \rightarrow X_{s} \gamma$ branching fraction in which the effects of a photon-energy cut $E_{\gamma} \geq 1.6 \mathrm{GeV}$ have been properly taken into account. Low-scale perturbative corrections lower the prediction for the branching ratio and introduce a significant theoretical uncertainty even at NNLO. Our result is about 1.4 standard deviations lower than the world-average experimental value. This re-opens the door for explorations of New Physics contributions to rare flavor-changing $B$-decay processes.

Acknowledgments: The research of T.B. was supported by the Department of Energy under Grant DEAC02-76CH03000. The research of M.N. was supported by the National Science Foundation under Grant PHY0355005. Fermilab is operated by Universities Research Association Inc., under contract with the U.S. Department of Energy.
[1] M. Misiak and M. Steinhauser, Nucl. Phys. B 683 (2004) 277 hep-ph/0401041.

[2] M. Gorbahn and U. Haisch, Nucl. Phys. B 713 (2005) 291 hep-ph/0411071.

[3] M. Gorbahn, U. Haisch and M. Misiak, Phys. Rev. Lett. 95 (2005) 102004 hep-ph/0504194.

[4] M. Czakon, U. Haisch and M. Misiak, in preparation.

[5] K. Bieri, C. Greub and M. Steinhauser, Phys. Rev. D 67 (2003) 114019 hep-ph/0302051.

[6] I. Blokland et al., Phys. Rev. D 72 (2005) 033014 hep-ph/0506055.

[7] H. M. Asatrian et al., Nucl. Phys. B 749 (2006) 325 hep-ph/0605009.

[8] M. Misiak and M. Steinhauser, hep-ph/0609241

[9] M. Misiak et al., hep-ph/0609232

[10] O. Buchmüller and H. Flächer, Phys. Rev. D 73, 073008 (2006) hep-ph/0507253.

[11] E. Barberio et al. [Heavy Flavor Averaging Group (HFAG)], hep-ex/0603003

[12] K. Melnikov and A. Mitov, Phys. Lett. B 620 (2005) 69 hep-ph/0505097.

[13] H. M. Asatrian et al., hep-ph/0607316

[14] M. Neubert, Eur. Phys. J. C 40, 165 (2005) hep-ph/0408179.
[15] T. Becher and M. Neubert, Phys. Lett. B 633, 739 (2006) hep-ph/0512208.

[16] T. Becher and M. Neubert, Phys. Lett. B 637, 251 (2006) hep-ph/0603140.

[17] S. J. Lee, M. Neubert and G. Paz, hep-ph/0609224

[18] G. P. Korchemsky and G. Sterman, Phys. Lett. B 340 , 96 (1994) hep-ph/9407344.

[19] M. Neubert, Phys. Rev. D 49, 3392 (1994) hep-ph/9311325; Phys. Rev. D 49, 4623 (1994) hep-ph/9312311.

[20] T. Becher and M. Neubert, Phys. Rev. Lett. 97, 082001 (2006) hep-ph/0605050.

[21] M. Neubert, Phys. Rev. D 72, 074025 (2005) hep-ph/0506245.

[22] T. Becher and M. Neubert, in preparation.

[23] Z. Ligeti, M. E. Luke, A. V. Manohar and M. B. Wise, Phys. Rev. D 60, 034019 (1999) hep-ph/9903305.

[24] S. W. Bosch, B. O. Lange, M. Neubert and G. Paz, Nucl. Phys. B 699, 335 (2004) hep-ph/0402094.

[25] M. Neubert, Phys. Lett. B 612, 13 (2005) hep-ph/0412241.

[26] M. B. Voloshin, Phys. Lett. B 397, 275 (1997) hep-ph/9612483. 